\title{
Evaluación de parámetros productivos en porcinos faenados con diferentes pesos
}

\author{
Rodríguez, I.; Leiva, A.; González, D.; Mesa, A. \\ Departamento de Ciencias Morfológicas, Facultad de Ciencias Veterinarias, \\ Universidad Nacional de Asunción, San Lorenzo, Paraguay. \\ E-mail: mrodriguez@vet.una.py
}

\begin{abstract}
Resumen
Rodríguez, I.; Leiva, A.; González, D.; Mesa, A.: Evaluación de parámetros productivos en porcinos faenados con diferentes pesos. Rev. Vet. 32:1, 84-88, 2021. Esta investigación se desarrolló en el Departamento de Itapúa, Paraguay, con el objeto de evaluar parámetros productivos en la línea porcina obtenida del cruce entre Large White y Landrace, faenados con diferentes pesos. Fueron analizados el peso vivo (PV), peso de la canal caliente (PCC), peso de la canal fría (PCF), rendimiento de la canal caliente (RCC), rendimiento de la canal fría (RCF) y merma (M), así como los pesos de las vísceras torácicas (PVT) y abdominales (PVA) de un total de 6.476 animales agrupados en 21 lotes, procedentes de seis granjas proveedoras incorporadas al sistema integrado de producción. Cada lote estuvo constituido por tres categorías clasificadas por el peso de la canal caliente en: categoría uno (hasta $73 \mathrm{~kg}$ ), categoría dos (de 73,1 hasta $79 \mathrm{~kg}$ ) y categoría tres (más de $79 \mathrm{~kg}$ ). Los datos se analizaron con el software estadístico $R$. Mediante el test de Kolmogorov-Smirnov se verificó la normalidad de la distribución de las observaciones, luego fueron estimadas medidas de tendencia central y dispersión, aplicando análisis de varianza con diseño de bloques incompletos balanceados y test de Tukey como prueba post hoc. Se detectaron diferencias significativas en cuanto al PV, PCC y PCF, destacándose la categoría tres con los registros más altos. En cuanto a los RCC y RCF se distinguieron a los grupos dos y tres como los de mejor desempeño, con idéntico porcentaje de pérdida por evaporación tras la refrigeración; mientras que la categoría uno tuvo menor performance en todos los parámetros analizados. Dos proveedores se caracterizaron por el suministro de animales con el mayor promedio de PV, y solo un proveedor proporcionó animales más ligeros, aunque con mayores RCC y RCF. El peso de las vísceras no mostró diferencias significativas entre proveedores $(\mathrm{p}<0,05)$.
\end{abstract}

Palabras clave: cerdos, canal, merma, rendimiento, vísceras.

\begin{abstract}
Rodríguez, I.; Leiva, A.; González, D.; Mesa, A.: Evaluation of productive parameters in fished pigs with different weights. Rev. Vet. 32: 1, 84-88, 2021. Research was conducted in the department of Itapúa, Paraguay. The objective was to evaluate productive parameters in the porcine line obtained from the crossing between Large White and Landrace, fished with different weights. The following was analyzed: live weight (LW), hot carcass weight (HCW), cold carcass weight (CCW), hot carcass yield ( $\mathrm{HCY}$ ), cold carcass yield (CCY) and depletion (D), as well as the weights of the thoracic (TVW) and abdominal viscera (AVW) from a total of 6,476 animals grouped in 21 batches, coming from six supplier farms incorporated to the integrated system of production. Each batch was formed by three categories classified by the hot carcass weight in: category one, until $73 \mathrm{~kg}$; category two, from 73.1 to $79 \mathrm{~kg}$, and category three, with more than $79 \mathrm{~kg}$. Data were analyzed with $R$ statistical software; through the Kolmogorov-Smirnov test it was verified the normality in the distribution of the observations. Then, measures of central tendency and dispersion were estimated, applying variance analysis with balanced incomplete block design and Tukey test as a post-hoc test. Significant differences were detected in terms of $\mathrm{LW}, \mathrm{HCW}, \mathrm{CCW}$, standing out category three with the highest registers. As for the $\mathrm{HCY}$ and $\mathrm{CCY}$, groups two and three were the ones with the best performance, with identical percentage of evaporation loss, after refrigeration; whereas, category one had the lowest performance in all the analyzed parameters. Two suppliers were characterized by the provision of animals with the highest average of LW; only one supplier
\end{abstract}


provided lighter animals, although the HCY and CCY were higher. The viscera weight did not show significant differences among suppliers $(\mathrm{p}<0,05)$.

Key words: pigs, carcass, depletion, yield, visceras.

\section{INTRODUCCIÓN}

Inicialmente, la cría de cerdos estuvo encaminada a satisfacer la demanda de grasa y carne. En la actualidad se orienta principalmente a la producción de carne proveniente de animales magros y mejorados genéticamente, con ventajas competitivas en relación a otros tipos de carne por su valor nutritivo y palatabilidad ${ }^{1,10}$.

A nivel mundial, la producción porcina ha tenido un crecimiento sostenido, siendo China, la Unión Europea y Estados Unidos responsables de más del 86\% de la producción mundial de cerdos ${ }^{12}$.

En la región, el sector se encuentra en auge y Paraguay no es la excepción. En el año 2018 fueron faenados 587.108 cerdos, exportando carne, menudencias y despojos a países como Rusia, Vietnam y Angola. Por otro lado, a nivel local, la preferencia por la carne de origen porcino ocupa el tercer lugar, con un consumo per cápita de cinco $\mathrm{kg} / \mathrm{añono}^{6,18}$.

En cuanto a la producción, es menester destacar que su propósito es la obtención de un producto de calidad y rendimiento superiores; así, las características de la canal y el rendimiento en carne comercializable son criterios fundamentales para la mayoría de los eslabones de la cadena productiva ${ }^{1}$.

La canal porcina rinde más del $75 \%$ de su peso vivo, debido a que su aparato digestivo es poco voluminoso, pudiendo alcanzar incluso $85 \%$. No obstante, la demanda está dirigida a un animal joven, de $100 \mathrm{~kg}$ que ofrezca entre $75 \%$ y $79 \%$ de rendimiento en la canal ${ }^{17}$.

$\mathrm{El}$ rendimiento tiene interés tanto para el productor como para el frigorífico. En este sentido, debe existir una estrecha relación entre el peso de los animales, que de-termina su precio de compra, y el rendimiento de los mismos ${ }^{1}$. En ocasiones, esto se ve enmascarado por adulteraciones en los pesos que van desde excesos de comidas antes de la venta, pesajes incorrectos o compra por apreciación en base a criterios subjetivos, lo cual ocasiona falta de correspondencia entre los resultados esperados y la realidad objetiva, con consecuentes pérdidas para la industria ${ }^{9}$.

En este contexto, la determinación de ciertos parámetros productivos en cerdos con diferentes pesos de faena, pretende contribuir con información técnica y científica necesaria para orientar la toma de decisiones y como herramienta válida para la eva-luación de resultados.

De esta manera, la investigación tuvo como objetivo, evaluar parámetros productivos en la línea porcina obtenida del cruce entre Large White y Landrace, faenada con diferentes pesos.

\section{MATERIAL Y MÉTODOS}

La investigación se llevó a cabo en el Distrito de Fram, ubicado en el Departamento de Itapúa (Paraguay), a $27^{\circ} 11^{\prime} 33,09^{\prime \prime}$ latitud sur, 56 $00^{\circ} 51,17^{\prime \prime}$ longitud oeste, y a $118 \mathrm{~m}$ sobre el nivel del mar. Situado en la región del bosque atlántico del Alto Paraná, registra precipitaciones pluviales entre 1.600 y $1.700 \mathrm{~mm}$ por año y una temperatura promedio anual de $21^{\circ} \mathrm{C}$. Constituye uno de los distritos con mayor ingreso per cápita a nivel país, siendo sus principales rubros económicos la agricultura, agroindustria y ganadería ${ }^{3,7}$.

Los datos fueron obtenidos durante el mes de junio de 2019 a partir de canales procedentes de un cruce comercial entre porcinos Large White y Landrace, sin distinción de sexo, incluyendo hembras enteras, machos castrados quirúrgicamente y machos inmunocastrados. Todos los animales contaban con 140 días de edad y fueron sometidos a un periodo de engorde de 110 días en seis granjas proveedoras incorporadas al sistema integrado de producción.

Una vez en el frigorífico, tras cumplir un período de espera de 12 horas con libre acceso al agua, fueron pesados y posteriormente sacrificados observando el cumplimiento irrestricto de la Ley 4.840/13 de protección y bienestar animal vigente en el país. Posteriormente se registraron los pesos de las dos medias canales con el objetivo de calcular el rendimiento de la canal caliente. Finalmente, las canales fueron llevadas a una cámara fría a $4^{\circ} \mathrm{C}$ y $85 \%$ de humedad relativa, siendo pesadas 24 horas después para determinar el rendimiento de la canal fría ${ }^{9}$.

Las variables evaluadas fueron peso vivo (PV), peso de la canal caliente (PCC), peso de la canal fría (PCF), rendimiento de la canal caliente (RCC), rendimiento de la canal fría (RCF) y merma (M), así como los pesos de las vísceras torácicas (PVT) y abdominales (PVA), procedentes de 6.476 animales agrupados en 21 lotes.

Las canales de cada lote fueron clasificadas en tres categorías en función al peso de la canal caliente en: categoría 1 hasta $73 \mathrm{~kg}$; categoría 2 de 73,1 hasta $79 \mathrm{~kg}$; y categoría 3 con más de $79 \mathrm{~kg}$. En cuanto al peso de las vísceras, fueron separadas en rojas (corazón y pulmones) y verdes (estómago, hígado, bazo e intestinos), pesadas por lote, constituyendo así cada uno de ellos una unidad observacional.

Los datos obtenidos fueron analizados estadísticamente con el software $R^{14}$. En primer lugar, se verificó la distribución teórica de las observaciones con el test de Kolmogorov-Smirnov; posteriormente se calcularon medidas de tendencia central y dispersión, aplicando luego análisis de varianza con diseño de bloques in- 
completos balanceados, donde los bloques representaron a cada uno de los proveedores. Se utilizó el test de Tukey como prueba post hoc.

\section{RESULTADOS Y DISCUSIÓN}

La distribución de las observaciones clasificadas por categoría y proveedores, se observa en la Figura 1, destacándose una mayor cantidad de animales agrupados en la categoría tres, vale decir con pesos de la canal caliente superiores a $79 \mathrm{~kg}$.

Cabe destacar que los grupos de observación estuvieron constituidos en su totalidad por cerdos de la misma edad y línea comercial, sin distinción de sexo. En ese sentido, bajo condiciones de cría extensiva, no fue detectado el efecto sexo sobre el rendimiento, calidad de la carne o perfil de los ácidos grasos de la grasa subcutánea ${ }^{15}$. No obstante, se han referido discrepancias que podrían estar vinculadas con las diferencias en cuanto al método utilizado al momento de la faena ${ }^{11}$. El análisis de la distribución reveló que los datos referentes al peso vivo y peso de la canal caliente y fría, se ajustaron a la normal conforme se observa en la Figura 2.

En cuanto a las medidas de resumen (Tabla 1), el comportamiento de las variables productivas mostró valores superiores para animales de la categoría tres, junto a mayores dispersiones en torno a la media sobre
Tabla 1. Medidas de tendencia central y dispersión de variables productivas evaluadas en tres categorías de cerdos con diferentes pesos.

\begin{tabular}{ccccccc}
\hline variables & unidad & categorías & $\mathrm{n}$ & $\bar{x}$ & $\pm \mathrm{DE}$ & $\mathrm{CV}$ \\
\hline \multirow{3}{*}{$\mathrm{PV}$} & \multirow{2}{*}{$\mathrm{kg}$} & 2 & 771 & 90,52 & 9,33 & 10,30 \\
& & 3 & 4803 & 103,36 & 3,03 & 2,93 \\
& & 1 & 771 & 66,65 & 6,70 & 10,05 \\
$\mathrm{PCC}$ & $\mathrm{kg}$ & 2 & 902 & 76,26 & 1,70 & 2,22 \\
& & 3 & 4803 & 90,59 & 7,63 & 8,42 \\
& & 1 & 771 & 64,39 & 6,55 & 6,55 \\
$\mathrm{PCF}$ & $\mathrm{kg}$ & 2 & 902 & 73,77 & 1,80 & 1,80 \\
& & 3 & 4803 & 87,64 & 7,42 & 7,42 \\
& & 1 & 771 & 73,66 & 1,45 & 1,97 \\
$\mathrm{RCC}$ & $\%$ & 2 & 902 & 73,81 & 1,45 & 1,96 \\
& & 3 & 4803 & 74,21 & 1,33 & 1,79 \\
& & 1 & 771 & 71,16 & 1,48 & 2,08 \\
$\mathrm{RCF}$ & $\%$ & 2 & 902 & 71,39 & 1,51 & 2,12 \\
& & 3 & 4803 & 71,80 & 1,48 & 2,06 \\
& & 1 & 771 & 2,51 & 0,74 & 29,54 \\
$\mathrm{M}$ & $\%$ & 2 & 902 & 2,41 & 0,73 & 30,06 \\
& & 3 & 4803 & 2,41 & 0,77 & 31,87 \\
\hline
\end{tabular}

PV: peso vivo; PCC: peso canal caliente; PCF: peso canal fría; RCC: rendimiento canal caliente; RCF: rendimiento canal fría; M: merma; $\mathrm{kg}$ : kilogramos; \%: porcentaje; $\mathrm{n}$ : cantidad de animales; $\bar{x}$ :promedio; $\pm \mathrm{DE}$ : desvío estándar; CV: coeficiente de variación.

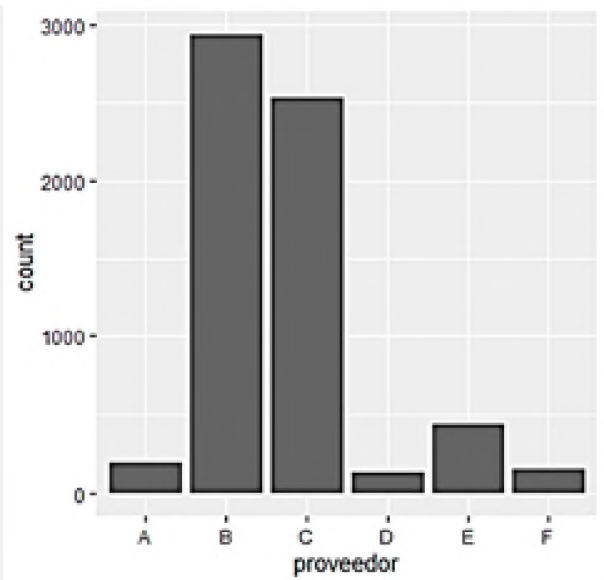

Figura 1. Distribución de las unidades observacionales clasificadas por categoría y proveedor.
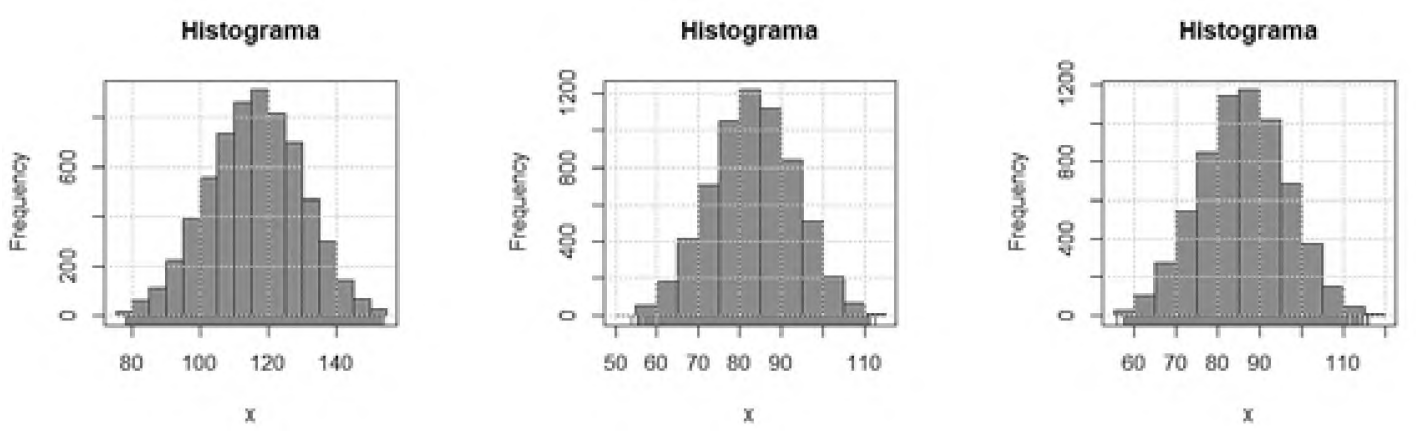

Figura 2. Distribución normal de las variables peso vivo (A), peso de la canal caliente (B) y fría (C), en animales con diferentes pesos de faena. 
todo en cuanto al peso vivo, peso de la canal caliente y fría, seguidos por los de la categoría uno.

Este comportamiento pudo responder al hecho que no existieron pesos mínimo y máximo establecidos como requisito para la faena de los animales; no obstante, se evidenció una notable uniformidad en las variables productivas; a excepción de la variabilidad registrada en cuanto a pérdidas por refrigeración, que resultó ser alta en todas las categorías.

El diseño de bloques incompletos balanceados proveyó estimaciones de medias de cada categoría. Los resultados del test de comparación de medias se observan en la Tabla 2.

El comportamiento en cuanto al peso vivo y peso de la canal caliente y fría, fue estadísticamente diferente en las tres categorías $(\mathbf{p}<0,05)$, destacándose el número tres con los mayores registros. Estos resultados son congruentes con el hecho que los grupos fueron establecidos en base al peso de la canal caliente.

Tabla 2. Comparación de medias de parámetros productivos obtenidos a partir de cerdos con diferentes pesos.

\begin{tabular}{ccccccc}
\hline categorías & PV $(\mathrm{kg})$ & PCC $(\mathrm{kg})$ & $\mathrm{PCF}(\mathrm{kg})$ & $\mathrm{RCC}(\%)$ & $\mathrm{RCF}(\%)$ & M (\%) \\
\hline 1 & $91,61_{\mathrm{a}}$ & $68,31_{\mathrm{a}}$ & $66,04_{\mathrm{a}}$ & $74,41_{\mathrm{a}}$ & $71,91_{\mathrm{a}}$ & $2,50_{\mathrm{b}}$ \\
2 & $104,20_{\mathrm{b}}$ & $77,72_{\mathrm{b}}$ & $75,22_{\mathrm{b}}$ & $74,55_{\mathrm{b}}$ & $72,14_{\mathrm{b}}$ & $2,41_{\mathrm{a}}$ \\
3 & $120,79_{\mathrm{c}}$ & $90,07_{\mathrm{c}}$ & $87,17_{\mathrm{c}}$ & $74,60_{\mathrm{b}}$ & $72,20_{\mathrm{b}}$ & $2,41_{\mathrm{a}}$ \\
\hline
\end{tabular}

Medias con letras diferentes por columnas son estadísticamente significativas; PV: peso vivo; PCC: peso canal caliente; PCF: peso canal fría; RCC: rendimiento canal caliente; RCF: rendimiento canal fría; $\mathrm{M}$ : merma; $\mathrm{kg}$ : kilogramos; \%: porcentaje.

Tabla 3. Comparación de medias de parámetros productivos en cerdos procedentes de diferentes proveedores.

\begin{tabular}{ccccccc}
\hline proveedor & PV $(\mathrm{kg})$ & PCC $(\mathrm{kg})$ & PCF $(\mathrm{kg})$ & RCC $(\%)$ & RCF $(\%)$ & M (\%) \\
\hline A & $110,52_{\mathrm{c}}$ & $82,97_{\mathrm{c}}$ & $80,46_{\mathrm{c}}$ & $74,93_{\mathrm{c}}$ & $72,63_{\mathrm{c}}$ & $2,30_{\mathrm{a}}$ \\
B & $104,15_{\mathrm{b}}$ & $76,29_{\mathrm{a}}$ & $73,79_{\mathrm{a}}$ & $73,30_{\mathrm{a}}$ & $70,89_{\mathrm{a}}$ & $2,41_{\mathrm{a}}$ \\
C & $109,54_{\mathrm{c}}$ & $81,65_{\mathrm{c}}$ & $78,95_{\mathrm{c}}$ & $74,47_{\mathrm{b}}$ & $72,00_{\mathrm{b}}$ & $2,47_{\mathrm{a}}$ \\
D & $104,36_{\mathrm{b}}$ & $76,45_{\mathrm{a}}$ & $73,93_{\mathrm{a}}$ & $73,30_{\mathrm{a}}$ & $70,88_{\mathrm{a}}$ & $2,42_{\mathrm{a}}$ \\
E & $100,83_{\mathrm{a}}$ & $76,68_{\mathrm{ab}}$ & $73,98_{\mathrm{a}}$ & $75,93_{\mathrm{e}}$ & $73,24_{\mathrm{d}}$ & $2,68_{\mathrm{b}}$ \\
F & $103,80_{\mathrm{b}}$ & $78,18_{\mathrm{b}}$ & $75,76_{\mathrm{b}}$ & $75,20_{\mathrm{d}}$ & $72,84_{\mathrm{c}}$ & $2,36_{\mathrm{a}}$ \\
\hline
\end{tabular}

Medias con letras diferentes por columnas son estadísticamente significativas; PV: peso vivo; PCC: peso canal caliente; PCF: peso canal fría; RCC: rendimiento canal caliente; RCF: rendimiento canal fría; $\mathrm{M}$ : merma; $\mathrm{kg}$ : kilogramos; \%: porcentaje.
En ese sentido, el peso de la canal de un cerdo reentiono-entorno en la que convergen factores genéticos y sistemas de manejo em-

En cuanto a los rendimientos de la canal caliente y fría, la categoría 1 registró valores inferiores, constadose un aumento en función al incremento del peso Contratam con diferentes pesos de faena fueron reportados criollo pelón ${ }^{16}$ ese sentido, se han referido mermas asociadas al sistema de producción, siendo mayor en sistemas extensivos; sin embargo, en este estudio y bajo sistemas de producción intensiva, todas las categorías registraron valores superiores a las reportadas en otras investigaciones 8,13

En este punto es importante señalar que los porcentajes de merma junto a otros factores, influyen en la calidad y cambios de la carne del estado normal a la condición PSE (pálida, suave y exudativa) entre 45 minutos y 24 horas post-mortem. En el mismo orden de ideas, se han reportado mayores pérdidas por mermas en canales con $\mathrm{pH}$ bajos, descenso congruente con la condición PSE, mediada por la sensibilidad de algunos cerdos al estrés y otras condicionantes como densidad de transporte, tipo de alimento, tipo de camión y tiempo de reposo ${ }^{5,13}$.

Los hallazgos podrían suponer diferencias en cuanto al nivel

Tabla 4. Promedio del peso de las vísceras de cerdos, estimada en kg (kilogramos) y procedentes de diferentes proveedores.

\begin{tabular}{ccc}
\hline proveedor & peso vísceras rojas & peso vísceras verdes \\
\hline A & $4,399_{\mathrm{a}}$ & $9,78_{\mathrm{a}}$ \\
B & $3,84_{\mathrm{a}}$ & $8,45_{\mathrm{a}}$ \\
C & $4,299_{\mathrm{a}}$ & $9,45_{\mathrm{a}}$ \\
D & $3,68_{\mathrm{a}}$ & $8,19_{\mathrm{a}}$ \\
E & $3,49 \mathrm{a}$ & $7,80_{\mathrm{a}}$ \\
F & $3,68_{\mathrm{a}}$ & $8,21_{\mathrm{a}}$ \\
\hline
\end{tabular}

Medias con letras diferentes son estadísticamente significativas. de exposición a dichos factores; sin embargo, los resultados no pueden ser concluyentes en ese sentido, pues no se realizaron mediciones de los mismos o del $\mathrm{pH}$ de la carne.

Los promedios analizados por proveedores se observan en la Tabla 3. En cuanto a los pesos vivos de la canal caliente y fría, los más elevados correspondieron al proveedor A y no difirieron desde el punto de vista estadístico del C. Es importante acotar que ambos proveedores suministraron, en más del $90 \%$, animales de la categoría tres, situación que explicaría estos resultados. Por su parte, el proveedor E se caracterizó por provisión de animales con el promedio de peso vivo más 
bajo, rendimientos de la canal caliente y fría superiores, y mayor porcentaje de merma $(p<0,05)$.

El análisis del peso de las vísceras discriminadas en rojas (corazón y pulmones) y verdes (estómago, hígado, bazo e intestinos) determinó la existencia de similitud estadística (Tabla 4), lo que resulta coherente al considerar que se trata de animales que pertenecen en su totalidad a una línea comercial destinada a la producción de tejido magro, la que en general presenta órganos más grandes y pesados; en contrario, cerdos con menor capacidad muscular, tienen menos desarrollados los tejidos y sobre todo los órganos asociados al metabolismo y distribución de los aminoácidos ${ }^{16}$.

Pese a las semejanzas verificadas es importante destacar que, a diferencia del proveedor $\mathrm{E}$, los proveedores A y $\mathrm{C}$ mostraron pesos viscerales numéricamente superiores, en consonancia con el hecho que estos últimos proporcionaron una cantidad importante de animales de la categoría tres.

En ese sentido, a mayor peso vivo, mayor peso de los órganos torácicos y abdominales, excepto ciego e intestino delgado, que muestran menos desarrollo debido a que la canal crece más rápidamente que el intestino, que representa una proporción menor del animal ${ }^{4}$.

En base a los resultados obtenidos se concluye que, para el sistema integrado de producción evaluado, con cruces comerciales obtenidos a partir de Large White por Landrace y bajo condiciones de manejo intensivo, animales de las categorías dos y tres resultan los adecuados en cuanto a indicadores productivos de peso y rendimiento, destacándose una aceptable uniformidad en dichas características.

Agradecimientos. Al Programa Universitario de Becas Andrés Borgognon Montero por el financiamiento y a la Unión de Productores de Itapúa por permitir la realización de este estudio.

\section{REFERENCIAS}

1. Balseca MA, Bello LD. 2014. Desempeño de cerdos de engorde con dos programas comerciales de alimentación. Tesis de grado, Escuela Agrícola Panamericana, Zamorano, Honduras, p. 25.

2. Barlocco N, Vadell A, Franco J 2000. Características de carcasas de cerdos con diferente proporción de genes Pampa, Duroc y Large White. Memorias XVI Reunión Latinoam. Prod. Anim., Montevideo, Uruguay.

3. Bartrina L. 2007. Contexto geográfico general. En: Biodiversidad del Paraguay: una aproximación a sus realidades, $1^{\circ}$ ed., Fundación Moisés Bertoni, Asunción, p. 25-32.

4. Casas G, Afanador G, Rodríguez D. 2009. Componentes anatómicos y coeficientes alométricos en cerdos machos castrados desde el nacimiento. Rev Colomb Cienc Pecu 22: 156-167.

5. Castrillon W, Fernández J, Restrepo L. 2007. Variables asociadas con la presentación de carne PSE (pálida, suave, exudativa) en canales de cerdo. Rev Col Cienc Pecu 20 : 327-338.

6. Chuen M, Centurión C, Corrales MP, González E. 2015. Manual de producción porcina. Min. Agric. Ganad. \& Univ. Asunción, Paraguay, p. 85.

7. DGEEC. 2003. Atlas censal del Paraguay. Disponible en https://www.dgeec.gov.py/Publicaciones/Biblioteca/Atlas Censal del Paraguay/10 Atlas Itapua censo.pdf

8. Fernández M et al. 2014. Efecto del cruce sobre las características de la canal de cerdo celta sacrificado a 7 meses. Actas Iberoam de Conserv Anim 4: 144-146.

9. García A et al. 2012. Mermas y rendimientos en el proceso de sacrificio del ganado porcino. Rev Comput Prod Porcina 19: 148-151.

10. Guzmán JC, Sánchez E. 2001. Evaluación de las características tecnológicas ( $\mathrm{pH}$, color y marmoleo) de calidad de la carne de cerdo con relación a tres diferentes sistemas de sacrificio. Tesis de grado, Univ. Guadalajara, Jalisco, México, p. 52

11. Latorre MA, García BE, Ariño L. 2008. The effects of sex and slaughter weight on growth performance and carcass traits of pigs intended for dry-cured ham from Teruel (Spain). J Anim Sci 86: 1933-1942.

12. Merchán JO. 2016. Estudio de factibilidad financiera para la implementación de un plantel porcino de engorde en la Comuna Dos Mangas, Santa Elena. Tesis de grado, Univ. Estat. Península de Santa Elena, Ecuador, p. 93.

13. Peinado B, Almela L, Duchi N, Poto A. 2009. Parámetros de calidad en la canal y en la carne de cerdo chato murciano. Eurocarne: https://eurocarne.com/revista

14. R Core Team 2018. R: a language and environment for statistical computing, 3.4.4. R. Foundation for Statistical Computing, Vienna, Austria.

15. Rodríguez JA. 2016. Calidad de la canal, la carne y la grasa de cerdos de cruce comercial, cebados al aire libre y sacrificados a pesos elevados. Tesis de Maestría, Univ Zaragoza, España, p. 81.

16. Santos R, Trejo W, Osorto H. 2011. Rendimiento de la canal y desarrollo de los órganos torácicos y abdominales de los 25 a los $45 \mathrm{~kg}$ en cerdos criollos pelones. Rev Cientif FCV-LUZ 21: 396-402.

17. Segarra EB, Salinas LR. 2016. Influencia de la edad, fenotipo, sexo y peso al sacrificio sobre los indicadores de calidad de los porcinos faenados en el Camal de Azogues. Tesis de grado, Universidad de Cuenca, Ecuador, p. 98.

18. SENACSA. 2018. Estadística pecuaria 2018. Disponible en:https:/www.senacsa.en https:/www.senacsa.gov.py/ index.php/informacion-publica/estadistica-pecuaria 